\title{
Etude des rendements en fromagerie de Camembert et de Saint-Paulin
}

\author{
par \\ J.-L. MAUBOIS, G. RICORDEAU et G. MOCQUOT \\ avec la collaboration technique de J.Y. DUPONT, E. GERVAIS \\ et N. BARBIER
}

Un industriel fromager doit être à même de prévoir le " rendement » en fromage du lait qu'il reçoit (on dit aussi la "valeur fromagère du lait ") c'est-à-dire la quantité de fromage qui peut être obtenue à partir d'une quantité définie de lait. Il y a à cela deux raisons principales : la première tient au fait que les produits fabriqués doivent être conformes à la législation en vigueur et doivent donc présenter une composition bien définie, la seconde tient à ce que la bonne marche d'une entreprise industrielle ne peut se concevoir sans une prévision du « rendement » de la matière première (lait) en produit fini (fromage). Une telle connaissance a priori du rendement permet, en effet, de réduire considérablement les marges de sécurité coûteuses que nécessitent l'hétérogénéité (d'un lieu et d'un jour à l'autre) de la matière première et celle des produits finis.

De nombreux travaux ont été consacrés, depuis plus de 75 ans, à " la valeur fromagère " du lait. Mais la plupart de ces études ont porté sur la transformation du lait en fromage du type pâte pressée ou cuite, essentiellement fromage de Cheddar (Mc Dowall, 1936 ; Shelton, 1945 ; Van Slyke et Price, 1952) et fromage d'Emmental (Schulz et Kay, 1957) ou Gruyère de Comté (Mocquot, Ricordeau et Auriol, 1963). Dans ces types de fromages, la teneur en eau du produit fini varie peu et il est relativement simple de mettre en évidence l'influence que peut avoir tel ou tel composant du lait de fabrication sur le rendement en fromage.

Il n'en est pas de même pour les fromages du type « pâte molle " où l'on constate une variation considérable de la teneur en eau des fromages d'une fabrication à l'autre, voire même d'un fromage à l'autre. Ces différences de teneur en eau entraînent des variations parallèles du rendement en poids de fromage frais ou en poids de fromage sec (Maubois et Mocquot, 1967). De ce fait, les relations existant entre les composants du lait de fabrication et le rendement en fromage peuvent être masquées par des différences d'égouttage 
dues à des facteurs bactériologiques ou technologiques que le fromager de pâte molle n'a pas su, ou n'a pas pu, contrôler.

Peu d'études ont été consacrées à ce type de fabrication. Mocquot, Blanc-Patin, Sainclivier, Rousseaux et Jeunet (1963) ont montré à partir de fabrications expérimentales, l'influence de la richesse du lait prise dans son ensemble, sur le rendement en fromage ; de plus ces mêmes auteurs, en triant les laits en laits " riches » et laits " pauvres ", ont tenté de définir le rôle joué par chacun des constituants : graisse d'une part, protéines d'autre part sur le rendement en fromage. Ricordeau et Mocquot (1967), Portmann Pierre et Vedrenne (1968) ont étudié la transformation du lait de chèvre en fromage et montré le rôle décisif joué par la matière azotée du lait dans le rendement en fromage.

L'objet de la présente étude a été de mettre en évidence les relations qui pouvaient exister dans des fabrications de fromages à l'échelle industrielle entre les différents composants du lait et le rendement en fromage de type Camembert d'une part et de type Saint-Paulin d'autre part. Pour ce faire, nous avons déterminé les paramètres caractéristiques de 120 fabrications de Camembert, en vraie grandeur (10000 l chacune) et de 122 fabrications de SaintPaulin (4800 1 chacune), réalisées dans des usines laitières de la région de Rennes. Puis nous avons éliminé par le calcul selon une formule que nous avions précédemment mise au point (Maubois et Mocquot, 1967) les variations considérables (coefficient de variation voisin ou supérieur à 5 p. 100) de teneur en eau des fabrications. Nous avons, alors, pu mettre en évidence le rôle joué par les composants du lait non seulement sur le plan quantitatif (poids du fromage obtenu) mais aussi sur le plan qualitatif (composition du fromage obtenu). Enfin, nous avons utilisé les résultats statistiques de notre étude pour proposer une méthode de prédétermination du rendement en fromagerie de Camembert et de Saint-Paulin.

\section{MATERIEL ET METHODES}

\section{1) Fabrications fromagères}

Nous avons suivi les méthodes de fabrications habituelles de trois usines du département d'Ille-et-Vilaine (A - B - C) en procédant de la manière suivante :

- Usine A: Cette usine fabrique quotidiennement en moyenne 5000 Camemberts à 45 p. 100 de matière grasse dans l'extrait sec, à partir de deux " tirées " d'environ $5000 \mathrm{l}$ de lait cru (additionné de ferments lactiques seulement durant la période hivernale). Pour chacune de nos 80 expérimentations, nous avons isolé une fraction aliquote de 6001 dont nous avons suivi la transformation en fromage. 
3001 de lait mesurés au double décalitre, après réchauffage à $30^{\circ}-35^{\circ} \mathrm{C}$ selon l'acidité étaient isolés de la première « tirée » de $5000 \mathrm{l}$ et versés dans 3 bassines de 100 I. Dans chacune de ces bassines, on ajoutait $20 \mathrm{ml}$ de présure commerciale (force 1/10 000). La coagulation et le durcissement du caillé, se faisaient ensuite dans les conditions habituelles de l'usine. Le caillé était moulé à la louche sur une table d'égouttage portant 300 moules. Les mêmes opérations se répétaient lors de la deuxième "tirée ", chaque moule recevant alors le complément de caillé nécessaire à l'obtention d'un fromage de format Camembert.

Le lendemain de la fabrication (jour $\mathrm{J}+1$ ), le sérum et les fromages recueillis étaient pesés. Les fromages étaient ensuite salés, ensemencés avec une suspension de spores de Penicillium Caseicolum, mis au haloir dans les conditions habituelles de l'usine. pesés.

Sept jours après la fabrication jour $\mathrm{J}+7$ les fromages étaient

- Usine B : Cette usine fabrique également 5000 Camemberts/jour en lait cru additionné de ferments lactiques, mais le moulage est effectué au moyen d'un " multimoule ».

6001 de lait sont mesurés au double décalitre puis après réchauffage à une température comprise entre $30^{\circ} \mathrm{C}$ et $35^{\circ} \mathrm{C}$ (selon l'acidité) emprésurés en bassines de 1001 à raison de $25 \mathrm{ml}$ de présure commerciale pour $100 \mathrm{l}$. Environ $1 \mathrm{~h}$ après l'emprésurage, le caillé découpé en cubes de $3 \times 3 \times 3 \mathrm{~cm}$ était moulé en une seule fois sur une table d'égouttage portant 300 moules. Le sérum était recueilli comme dans l'usine A. Les pesées du sérum et des fromages se faisaient aux mêmes dates après la fabrication : $\mathrm{J}+1$ et $\mathrm{J}+7$. usine.

Quarante-quatre expérimentations ont été réalisées dans cette

- Usine C : Cette usine fabrique $3000 \mathrm{~kg}$ de Saint-Paulin/jour à partir de lait pasteurisé ensemencé.

48001 de lait soit la totalité du lait contenu dans une cuve de fabrication étaient transformés en Saint-Paulin selon la technique habituelle de l'usine. Les fromages obtenus étaient pesés $3 \mathrm{j}$ après la fabrication (jour $\mathrm{J}+3$ ).

Cent-vingt-deux expérimentations ont été réalisées.

\section{2) Prélèvement et conservation des échantillons pour l'analyse}

a) Echantillons de lait de fabrication

- Les échantillons de lait destinés à l'analyse étaient prélevés une fois toutes les additions faites, soit dans la cuve de standardisation (usine A), soit dans les bassines (usine B), soit dans la cuve de fabrication (usine $\mathrm{C}$ ), avant emprésurage. 
- Les échantillons de lait étaient refroidis immédiatement après le prélèvement à $20^{\circ} \mathrm{C}$ et on procédait aussitôt à la détermination de la teneur en graisse. Puis ils étaient refroidis et maintenus à $+4^{\circ} \mathrm{C}$ jusqu'au moment des autres déterminations qui se faisaient le lendemain au laboratoire. Une partie aliquote de ces échantillons $(60 \mathrm{ml})$ était additionnée de chlorure mercurique et servait à la détermination de la teneur en protéines.

\section{b) Echantillons de sérum}

Après un mélange soigneux des cuves recueillant le sérum (usines A et B), on prélevait un échantillon de sérum que l'on maintenait à basse température jusqu'au moment de l'analyse.

\section{c) Echantillons de fromages}

Pour les usines A et B, 15 fromages (1 par claie de 20) étaient prélevés au hasard après la pesée au jour $\mathrm{J}+7$.

Pour l'usine C, un demi-fromage de Saint-Paulin sur les 260-290 fromages de la fabrication était prélevé au jour $\mathrm{J}+3$.

\section{3) Déterminations analytiques}

Les échantillons de lait et de sérum étaient réchauffés à $20^{\circ} \mathrm{C}$ et soigneusement mélangés par agitation.

Les 15 fromages prélevés à $\mathrm{J}+7$ lors des essais réalisés dans les usines A et B étaient partagés en trois piles de 5 fromages chacune. Une tranche médiane était découpée sur l'ensemble de chacune des piles, écroûtée (environ $2 \mathrm{~mm}$ d'épaisseur de croûte étaient enlevés), finement divisée au couteau et analysée aussitôt.

Après écroûtage (entre 1 et $2 \mathrm{~mm}$ de croûte étaient enlevés), on découpait sur le demi-Saint-Paulin prélevé dans l'usine $C$, une tranche parallèle au diamètre, d'environ $150 \mathrm{~g}$. Cette tranche était finement divisée au couteau et analysée aussitôt.

\section{Détermination de la teneur en matières azotées}

La teneur en azote était déterminée par microkjeldahl.

La teneur en azote $\times 6,38$ donne la teneur en matières azotées.

La quantité de matières azotées coagulables (M.A.C.) par la présure était calculée selon les indications données par Ricordeau et Mocquot (1967) mais cette proportion a été calculée, à la fois, à partir de l'analyse du sérum recueilli en usine (M.A.C. usine) et à partir de l'analyse de sérum obtenu au laboratoire (M.A.C. labo) dans les conditions suivantes : $100 \mathrm{ml}$ du lait de fabrication, portés à $30^{\circ} \mathrm{C}$, étaient emprésurés $(0,02 \mathrm{ml}$ de présure commerciale), maintenus au bain-marie à $30^{\circ} \mathrm{C}$ pendant $90 \mathrm{mn}$ puis filtrés sur papier Whatman $\mathrm{n}^{\circ}$ 40. La teneur en azote du lactosérum ainsi obtenu était déterminée lorsque le volume écoulé était de l'ordre de $40 \mathrm{ml}$. La 
quantité de caséine précipitable à $\mathrm{pH}$ 4,6 étalt déterminée selon la méthode d'Aschaffenburg et Drewry (1958).

Détermination de la teneur en matière grasse Gerber.

Des laits et lactosérums : méthode acido-butyrométrique de

Des fromages : méthode officielle ( $\left.n^{\circ} 5\right)$ de la Fédération Internationale de Laiterie.

Détermination de la teneur en substance sèche

Des laits et lactosérums : méthode officielle française.

Des fromages : méthode officielle $\left(n^{\circ} 4\right)$ de la Fédération Internationale de Laiterie.

Détermination de la densité des laits et des lactosérums

A $20^{\circ} \mathrm{C}$ avec un lacto-densimètre vérifié.

\section{4) Expression des résultats}

Le rendement en fromage est représenté par le poids de fromage obtenu à partir de $100 \mathrm{l}$ de lait mis en œuvre.

Les variations de teneur en substance sèche totale des fromages ont été éliminées en utilisant la correction que nous avons précédemment proposée (Maubois et Mocquot, 1967), soit :

$$
R_{2}^{\prime}=R_{2} \times \frac{f-s}{f^{\prime}-s}
$$

$\mathrm{R}_{2}$ est le rendement en poids de fromage d'une teneur en substance sèche totale " $\mathrm{f}$ » d'une certaine fabrication.

$\mathrm{R}_{2}{ }_{2}$ est le rendement en poids de fromage de cette même fabrication mais à une teneur en substance sèche totale du fromage prise en référence " $f$ '».

s est la teneur en substance sèche totale du lactosérum.

Les fromages de type Camembert ont été « ramenés » à un extrait sec égal à 45 p. 100 (moyenne de toutes les fabrications) ; les fromages du type Saint-Paulin ont été « ramenés » à un extrait sec égal à 48,2 p. 100 (moyenne des 122 fabrications).

\section{5) Symboles}

Dans les tableaux, nous utiliserons les symboles suivants :

$\mathrm{TB}=$ taux butyreux du lait de fabrication en $\mathrm{g}$ de graisse par 1 de lait $(\mathrm{g} / \mathrm{l})$

$\mathrm{TP}=$ taux de protéines du lait en $\mathrm{g} / \mathrm{l}$

TC = taux de caséine isoélectrique du lait en $\mathrm{g} / \mathrm{l}$ 
$\mathrm{MAC}_{\imath}=$ taux de matières azotées coagulables déterminé au laboratoire en $\mathrm{g} / \mathrm{l}$

MAC $_{u}=$ taux de matières azotées coagulables déterminé en fabrication en $\mathrm{g} / \mathrm{l}$

EST = taux de substance sèche totale du lait en $\mathrm{g} / \mathrm{l}$

$\mathrm{ESD}=$ taux de substance sèche dégraissée du lait en $\mathrm{g} / 1$

$\mathrm{R}_{1} \quad=$ rendement en fromage au démoulage en $\mathrm{kg}$ pour $100 \mathrm{l}$ de lait

$\mathrm{R}_{2} \quad=$ rendement en fromage à $7 \mathrm{j}$ en $\mathrm{kg}$ pour $100 \mathrm{l}$ de lait

$\mathrm{R}_{2}^{\prime} \quad=$ rendement en fromage à $7 \mathrm{j}$ corrigé pour l'extrait sec en $\mathrm{kg} / 100 \mathrm{l}$

$\mathrm{g} \quad=$ teneur en graisse du fromage à $7 \mathrm{j}$ en $\mathrm{g} / 100 \mathrm{~g}$

$\mathrm{f}=$ teneur en substance sèche totale du fromage à $7 \mathrm{j}$ en $\mathrm{g} / 100 \mathrm{~g}$

$\mathrm{s}=$ teneur en substance sèche totale du lactosérum moyen en $\mathrm{g} / \mathrm{l}$

$\mathrm{Z}_{1} \quad=$ poids de fromage frais au démoulage par $\mathrm{kg}$ de matière grasse du lait mis en œuvre

$\mathrm{Z}_{2} \quad=$ poids de fromage à $7 \mathrm{j}$ par $\mathrm{kg}$ de matière grasse du lait mis en œuvre

$\mathrm{Z}_{2} \quad=$ poids de fromage à $7 \mathrm{j}$ par $\mathrm{kg}$ de matière grasse du lait mis en œuvre, corrigé pour l'extrait sec

$\mathrm{n} \quad=$ nombre d'expérimentations

$\bar{x} \quad=$ moyenne arithmétique

$\sigma \quad=$ écart-type

$\mathrm{CV}=$ coefficient de variation $=\frac{\sigma}{\bar{x}} \times 100$

$\mathrm{r} \quad=$ coefficient de corrélation

Dans les analyses de régression multiples

$\mathrm{F} \quad=$ test relatif à toutes les variables (variance due à la régression/variance résiduelle) avec $V_{1}=\mathrm{k}$ et $V_{2}=\mathrm{n}-\mathrm{k}-1$ ( $\mathrm{k}$ étant le nombre de variables indépendantes)

$\mathrm{R}^{2} \quad=$ carré du coefficient de corrélation

$\mathrm{s}_{\mathrm{r}} \quad=$ estimation de l'écart-type résiduel du rendement

$\mathrm{t}^{2}=$ test relatif à chaque coefficient de régression $\mathrm{b}_{\mathrm{i}}\left(\mathrm{t}^{2}=\mathrm{b}^{2}{ }_{\mathrm{i}} / \mathrm{s}^{2} \mathrm{~b}_{1}\right)$.

\section{RESULTATS}

\section{1) Paramètres statistiques (tab. 1)}

a) Moyennes

Le lait réceptionné à la laiterie A présente des teneurs en protéines, en extrait sec dégraissé, en caséine isoélectrique, en matières azotées coagulables par la présure (M.A.C.) légèrement supérieures à celles du lait réceptionné à la laiterie $\mathrm{B}$. Cependant la proportion de M.A.C. dans les matières azotées totales est légèrement supérieure 
TABLEAU 1. - Résultats d'ensemble

\begin{tabular}{|c|c|c|c|c|c|c|c|c|c|}
\hline & \multicolumn{3}{|c|}{ Laiterie A ( $\mathrm{n}=78)$} & \multicolumn{3}{|c|}{ Laiterie $B(n=42)$} & \multicolumn{3}{|c|}{ Laiterie C $(n=122)$} \\
\hline & $\bar{x}$ & $\sigma$ & $\mathrm{CV}$ & $\bar{x}$ & $\sigma$ & $\mathrm{CV}$ & $\bar{x}$ & $\sigma$ & $\mathrm{CV}$ \\
\hline TB & 28,86 & 1,11 & 3,81 & 27,81 & 0,82 & 2,95 & 26,07 & 1,23 & 4,72 \\
\hline TP & 35,17 & 0,87 & 2,47 & 34,17 & 0,87 & 2,55 & 34,97 & 1,00 & 2,86 \\
\hline TC & 27,24 & 0,77 & 2,83 & 26,58 & 0,85 & 3,20 & 27,82 & 1,00 & 3,59 \\
\hline $\mathrm{MAC}_{l}$ & 25,68 & 0,82 & 3,19 & 25,22 & 0,79 & 3,13 & 26,41 & 0,92 & 3,48 \\
\hline $\mathrm{MAC}_{u}$ & 26,04 & 0,70 & 2,69 & 25,36 & 0,81 & 3,19 & - & - & - \\
\hline EST & 120,97 & 0,16 & 0,13 & 118,16 & 0,19 & 0,16 & - & - & - \\
\hline ESD & 92,11 & 1,16 & 1,26 & 90,35 & 1,49 & 1,65 & - & - & - \\
\hline $\mathrm{TP} / \mathrm{TB}$ & 1,221 & 0,55 & 4,50 & 1,229 & 0,039 & 3,17 & 1,344 & 0,042 & 3,12 \\
\hline ESD/TB & 3,196 & 0,131 & 4,10 & 3,251 & 0,096 & 2,95 & - & - & - \\
\hline $\mathrm{TC} / \mathrm{TB}$ & 0,945 & 0,042 & 4,44 & 0,956 & 0,033 & 3,45 & 1,069 & 0,054 & 5,05 \\
\hline $\mathrm{MAC}_{l} / \mathrm{TB}$ & 0,891 & 0,042 & 4,71 & 0,907 & 0,032 & 3,53 & - & - & - \\
\hline $\mathrm{MAC}_{u} / \mathrm{TB}$ & 0,904 & 0,041 & 4,54 & 0,912 & 0,030 & 3,29 & - & - & - \\
\hline TC/TP & 77,44 & 1,23 & 1,59 & 77,78 & 0,96 & 1,23 & 79,55 & 0,24 & 0,30 \\
\hline $\mathrm{MAC}_{l} / \mathrm{TP}$ & 73,01 & 1,42 & 1,94 & 73,81 & 0,99 & 1,36 & 75,51 & 0,25 & 0,33 \\
\hline $\mathrm{MAC}_{u} / \mathrm{TP}$ & 74,01 & 0,92 & 1,24 & 74,22 & 0,77 & 1,04 & - & - & - \\
\hline $\mathrm{R}_{1}$ & 15,81 & 0,68 & 4,28 & 16,21 & 1,04 & 6,58 & - & - & - \\
\hline $\mathrm{R}_{2}$ & 14,25 & 0,77 & 5,40 & 14,88 & 1,00 & 6,72 & 12,01 & 0,56 & 4,66 \\
\hline $\mathrm{R}_{2}^{\prime}$ & 14,10 & 0,35 & 2,48 & 13,41 & 0,36 & 2,68 & 12,01 & 0,15 & 1,25 \\
\hline $\mathrm{Z}_{1}$ & 5,49 & 0,29 & 5,28 & 5,83 & 0,39 & 6,69 & - & - & - \\
\hline $\mathrm{Z}_{2}$ & 4,94 & 0,27 & 5,47 & 5,36 & 0,37 & 6,90 & 4,61 & 0,20 & 4,34 \\
\hline $\mathrm{Z}_{2}^{\prime}$ & 4,88 & 0,14 & 2,79 & 4,82 & 0,125 & 2,59 & 4,61 & 0,18 & 3,90 \\
\hline $\mathrm{g}$ & 20,92 & 1,46 & 6,98 & 18,65 & 1,51 & 8,10 & 20,22 & 1,09 & 5,39 \\
\hline $\mathrm{f}$ & 44,69 & 2,09 & 4,68 & 41,34 & 2,33 & 5,64 & 48,24 & 1,60 & 3,32 \\
\hline $\mathrm{s}$ & 65,88 & 1,05 & 1,59 & 66,29 & 0,79 & 1,19 & - & - & - \\
\hline
\end{tabular}




\begin{tabular}{|c|c|c|c|c|c|c|c|c|c|c|c|c|}
\hline & TB & TP & TC & $\mathrm{MAC}_{l}$ & $\mathrm{MAC}_{u}$ & EST & ESD & $\mathrm{R}_{1}$ & $\mathrm{R}_{2}$ & $\mathrm{R}_{2}^{\prime}$ & $\mathrm{g}$ & $\mathrm{f}$ \\
\hline TP & $\begin{array}{l}\text { A } 0,02 \\
\text { B } 0,37^{*} \\
\text { C } 0,23^{*}\end{array}$ & & & & & & & & & & & \\
\hline $\mathrm{TC}$ & $\begin{array}{l}0,13 \\
0,39^{* *} \\
0,30^{* *}\end{array}$ & $\begin{array}{l}0,83^{* *} \\
0,94^{* *} \\
0,98^{* *}\end{array}$ & & & & & & & & & & \\
\hline $\mathrm{MAC}_{l}$ & $\begin{array}{l}0.12 \\
0,36^{*} \\
0,26^{* *}\end{array}$ & $\begin{array}{l}0,80^{* *} \\
0,91^{*} \\
0,97^{* *}\end{array}$ & $\begin{array}{l}0,90^{* *} \\
0,94^{* *} \\
0,94^{* *}\end{array}$ & & & & & & & & & \\
\hline $\mathrm{MAC}_{u}$ & $\begin{array}{l}0,06 \\
0,45^{* *} \\
-\end{array}$ & $\begin{array}{c}0,88^{* *} \\
0,96^{* *} \\
-\end{array}$ & $\begin{array}{l}0,87^{* *} \\
0,94^{* *} \\
-\end{array}$ & $\begin{array}{l}0,86^{* *} \\
0,94^{* *} \\
-\end{array}$ & & & & & & & & \\
\hline EST & $\begin{array}{l}0,68^{* *} \\
0,66^{* *}\end{array}$ & $\begin{array}{l}0,44^{* *} \\
0,67^{* *}\end{array}$ & $\begin{array}{l}0,52^{* *} \\
0,70^{* *} \\
-\end{array}$ & $\begin{array}{l}0,51^{* *} \\
0,70^{* *} \\
-\end{array}$ & $\begin{array}{l}0,45^{* *} \\
0,71^{* *} \\
-\end{array}$ & & & & & & & \\
\hline ESD & $\begin{array}{r}-0,03 \\
0,29 \\
-\end{array}$ & $\begin{array}{l}0,59^{* *} \\
0,65^{* *} \\
-\end{array}$ & $\begin{array}{l}0,59^{\star *} \\
0,68^{* *} \\
-\end{array}$ & $\begin{array}{l}0,58^{* *} \\
0,69^{* *} \\
-\end{array}$ & $\begin{array}{l}0,55^{\star *} \\
0,65^{\star *} \\
-\end{array}$ & $\begin{array}{l}0,71^{* *} \\
0,91^{* *} \\
-\end{array}$ & & & & & & \\
\hline
\end{tabular}




\begin{tabular}{|c|c|c|c|c|c|c|c|c|c|c|c|c|}
\hline $\mathrm{R}_{1}$ & 0,16 & 0,09 & $0,22^{*}$ & $0,21^{*}$ & $0,27^{*}$ & $0,22^{*}$ & 0,15 & & & & & \\
\hline & $\begin{array}{c}0,22 \\
-\end{array}$ & $\begin{array}{c}0,48^{* *} \\
-\end{array}$ & $\begin{array}{l}0,53^{* *} \\
-\end{array}$ & $\begin{array}{l}0,47^{* *} \\
-\end{array}$ & $\begin{array}{c}0,48^{\star *} \\
-\end{array}$ & $\begin{array}{c}0,47^{\star \star} \\
-\end{array}$ & $\begin{array}{c}0,48^{\star \star} \\
-\end{array}$ & & & & & \\
\hline $\mathrm{R}_{2}$ & $\begin{array}{l}0,30^{\star \star} \\
0,19 \\
0,58^{\star \star}\end{array}$ & $\begin{array}{l}0,11 \\
0,45^{\star *} \\
0,70^{* *}\end{array}$ & $\begin{array}{l}0,29^{* *} \\
0,50^{* *} \\
0,73^{* *}\end{array}$ & $\begin{array}{l}0,30^{* *} \\
0,48^{* *} \\
0,71^{* *}\end{array}$ & $\begin{array}{l}0,28^{* *} \\
0,48^{* *} \\
-\end{array}$ & $\begin{array}{l}0,40^{* *} \\
0,49^{* *} \\
-\end{array}$ & $\begin{array}{l}0,25^{\star} \\
0,52^{* *} \\
-\end{array}$ & $\begin{array}{l}0,78^{* *} \\
0,76^{* *} \\
-\end{array}$ & & & & \\
\hline $\mathrm{R}_{2}^{\prime}$ & $\begin{array}{l}0,70^{\star \star} \\
0,60^{\star *} \\
0,58^{\star *}\end{array}$ & $\begin{array}{l}0,31^{\star \star} \\
0,38^{\star} \\
0,44^{\star *}\end{array}$ & $\begin{array}{l}0,17 \\
0,32^{\star} \\
0,46^{\star *}\end{array}$ & $\begin{array}{l}0,16 \\
0,37^{*} \\
0,44^{* *}\end{array}$ & $\begin{array}{l}0,18 \\
0,41^{* *} \\
-\end{array}$ & $\begin{array}{l}0,60^{* *} \\
0,61^{* *} \\
-\end{array}$ & $\begin{array}{l}0,15 \\
0,44^{\star \star} \\
-\end{array}$ & $\begin{array}{l}0,12 \\
0,20 \\
-\end{array}$ & $\begin{array}{l}0,13 \\
0,26 \\
-\end{array}$ & & & \\
\hline $\mathrm{g}$ & $\begin{array}{l}0,20 \\
0,16 \\
0,20^{*}\end{array}$ & $\begin{array}{l}-0,07 \\
-0,21 \\
-0,44^{* *}\end{array}$ & $\begin{array}{l}-0,27^{\star} \\
-0,30^{\star} \\
-0,43^{\star *}\end{array}$ & $\begin{array}{l}-0,26^{*} \\
-0,25 \\
-0,42^{\star *}\end{array}$ & $\begin{array}{c}-0,22^{*} \\
-0,23 \\
-\end{array}$ & $\begin{array}{c}-0,01 \\
-0,09 \\
-\end{array}$ & $\begin{array}{c}0,20 \\
-0,19 \\
-\end{array}$ & $\begin{array}{c}-0,55^{* *} \\
-0,63^{* *} \\
-\end{array}$ & $\begin{array}{l}-0,78^{* *} \\
-0,81^{* *} \\
-0,40^{* *}\end{array}$ & $\begin{array}{l}0,35^{* *} \\
0,29 \\
0,24^{*}\end{array}$ & & \\
\hline $\mathrm{f}$ & $\begin{array}{r}0,03 \\
0,04 \\
-0,10\end{array}$ & $\begin{array}{c}0,04 \\
-0,34^{* *} \\
-0,42^{* *}\end{array}$ & $\begin{array}{l}-0,21^{\star} \\
-0,43^{* *} \\
-0,44^{* *}\end{array}$ & $\begin{array}{l}-0,21^{*} \\
-0,38^{* *} \\
-0,43^{* *}\end{array}$ & $\begin{array}{c}-0,19 \\
-0,35^{\star *} \\
-\end{array}$ & $\begin{array}{c}-0,11 \\
-0,29 \\
-\end{array}$ & $\begin{array}{c}-0,18 \\
-0,39^{\star *} \\
-\end{array}$ & $\begin{array}{c}-0,68^{\star \star} \\
-0,70^{\star \star} \\
-\end{array}$ & $\begin{array}{l}-0,89^{\star *} \\
-0,91^{\star \star} \\
-0,57^{\star \star}\end{array}$ & $\begin{array}{l}0,33^{* *} \\
0,12 \\
0,25^{\star *}\end{array}$ & $\begin{array}{l}0,90^{* *} \\
0,91^{\star *} \\
-\end{array}$ & \\
\hline $\mathrm{s}$ & $\begin{array}{l}0,36^{* *} \\
0,15 \\
-\end{array}$ & $\begin{array}{l}0,09 \\
0,42^{* *} \\
-\end{array}$ & $\begin{array}{l}0,07 \\
0,45^{* *} \\
-\end{array}$ & $\begin{array}{l}0,08 \\
0,55^{* *} \\
-\end{array}$ & $\begin{array}{l}0,11 \\
0,47^{\star *} \\
-\end{array}$ & $\begin{array}{l}0,30^{* *} \\
0,49^{* *} \\
-\end{array}$ & $\begin{array}{l}0,06 \\
0,54^{* *} \\
-\end{array}$ & $\begin{array}{r}-0,02 \\
0,22 \\
-\end{array}$ & $\begin{array}{l}0,09 \\
0,27 \\
-\end{array}$ & $\begin{array}{l}0,21 \\
0,12 \\
-\end{array}$ & $\begin{array}{r}0,13 \\
-0,23 \\
-\end{array}$ & $\begin{array}{r}0,03 \\
-0,21 \\
-\end{array}$ \\
\hline
\end{tabular}

* significatif au seuil $P=0,05$

** significatif au seuil $\mathrm{P}=0,01$ 
dans le cas de la laiterie B $(74,2$ contre 74,0$)$. Pour une même usine, la détermination de cette proportion des M.A.C. dans les matières azotées totales à partir de l'analyse du lactosérum recueilli en fabrication donne une valeur supérieure (1 p. 100 pour la laiterie A, 0,4 p. 100 pour la laiterie $\mathrm{B}$ ) à celle qui peut être calculée à partir de l'analyse du lactosérum obtenu au laboratoire.

Les laiteries A et B utilisent pour leur fabrication un lait ayant à peu de choses près le même rapport TP/TB $(1,22$ contre 1,23$)$. Les rendements en fromage au démoulage et à $7 \mathrm{j}$ sont nettement plus élevés dans la laiterie B que dans la laiterie A $(16,2$ contre 15,8 et 14,9 contre 14,3) mais cela tient à ce que les fromages obtenus sont aussi plus humides (extrait sec 41,3 p. 100 contre 44,7 p. 100). A extrait sec égal, le rendement en fromage $\left(R_{2}^{\prime}\right)$ est plus faible dans la laiterie B. Ceci est confirmé par une teneur en substance sèche totale du lactosérum plus élevée.

La laiterie $\mathrm{C}$ ne fabrique pas le même type de fromage que les deux autres laiteries. Le lait utilisé est relativement moins riche en graisse $(\mathrm{TP} / \mathrm{TB}=1,34)$ et il subit un traitement thermique préalable, ce qui se traduit par une élévation de 2 p. 100 du taux de caséine et de la proportion des M.A.C. dans les matières azotées totales.

\section{b) Variabilité}

La teneur en protéines du lait de fabrication varie à peu près de la même manière dans les trois laiteries. La teneur en graisse (TB) varie un peu plus que le taux de protéines (TP) mais cette variation est à peu près inversement proportionnelle. Il résulte de ceci une teneur en substance sèche totale pratiquement invariable et une variabilité importante des rapports TP/TB, TC/TB, MAC $/$ TB et $\mathrm{MAC}_{n} / \mathrm{TB}$. La teneur en substance sèche dégraissée des laits mis en fabrication varie moins que le TP. Les proportions de caséine isoélectrique et de matières azotées coagulables dans les matières azotées totales des laits mis en œuvre varient peu dans les laiteries $A$ et $B$ et ne varient pratiquement pas dans la laiterie C.

Les rendements en fromage au démoulage $\left(R_{1}\right)$ et à 7 j $\left(R_{2}\right)$ sont nettement moins variables dans la laiterie A que dans la laiterie B. Ceci résulte probablement des différences dans le mode de fabrication. Le rendement en fromage au démoulage varie moins que le rendement à $7 \mathrm{j}$, notamment dans la laiterie $\mathrm{A}$. La correction par le calcul des différences de teneur en substance sèche totale des fromages (f) diminue considérablement la variabilité du rendement en fromage à $7 \mathrm{j}\left(\mathrm{R}_{2}^{\prime}\right)$.

La teneur en graisse du fromage apparaît comme l'élément le plus variable tant pour les fromages du type Camembert que pour les fromages du type Saint-Paulin.

La teneur en substance sèche totale des lactosérums de fabrication varie peu, sensiblement dans la même proportion que la teneur en substance sèche dégraissée du lait. 
TABLEAU 3

Matrice des corrélations entre les rendements $\mathrm{Z}$ et les rapports matière azotée/matière grasse

\begin{tabular}{|c|c|c|c|c|c|c|c|}
\hline & $\mathrm{Z}_{1}$ & $\mathrm{Z}_{2}$ & $Z_{2}^{\prime}$ & $\mathrm{TP} / \mathrm{TB}$ & TC/TB & $\mathrm{MAC}_{l} / \mathrm{TB}$ & $\mathrm{MAC}_{u} / \mathrm{TB}$ \\
\hline $\mathrm{Z}_{2}$ & $\begin{array}{c}0,80^{\star *} \\
0,77^{\star *} \\
-\end{array}$ & & & & & & \\
\hline $\mathrm{Z}_{2}^{\prime}$ & $\begin{array}{l}0,47^{\star *} \\
0,22 \\
-\end{array}$ & $\begin{array}{l}0,23^{*} \\
0,31^{*} \\
0,60^{* *}\end{array}$ & & & & & \\
\hline $\mathrm{TP} / \mathrm{TB}$ & $\begin{array}{c}0,54^{* *} \\
0,49^{* *} \\
-\end{array}$ & $\begin{array}{l}0,37^{\star *} \\
0,48^{\star *} \\
0,73^{\star *}\end{array}$ & $\begin{array}{l}0,79^{\star *} \\
0,49^{\star \star} \\
0,64^{\star *}\end{array}$ & & & & \\
\hline $\mathrm{TC} / \mathrm{TB}$ & $\begin{array}{c}0,56^{* *} \\
0,54^{* *} \\
-\end{array}$ & $\begin{array}{l}0,45^{\star *} \\
0,53^{\star *} \\
0,78^{\star \star}\end{array}$ & $\begin{array}{l}0,64^{\star *} \\
0,36^{* *} \\
0,62^{* *}\end{array}$ & $\begin{array}{l}0,94^{\star \star} \\
0,93^{\star \star} \\
0,98^{\star \star}\end{array}$ & & & \\
\hline $\mathrm{MAC}_{l} / \mathrm{TB}$ & $\begin{array}{c}0,54^{\star \star} \\
0,49^{\star \star} \\
-\end{array}$ & $\begin{array}{l}0,44^{\star *} \\
0,50^{\star *} \\
-\end{array}$ & $\begin{array}{c}0,61^{\star \star} \\
0,43^{\text {**}} \\
-\end{array}$ & $\begin{array}{l}0,91^{\star \star} \\
0,92^{\star \star} \\
-\end{array}$ & $\begin{array}{l}0,95^{\star \star} \\
0,94^{\star \star} \\
-\end{array}$ & & \\
\hline $\mathrm{MAC}_{u} / \mathrm{TB}$ & $\begin{array}{l}0,61^{\star \star} \\
0,48^{\star *} \\
-\end{array}$ & $\begin{array}{l}0,45^{\star \star} \\
0,50^{\star \star} \\
-\end{array}$ & $\begin{array}{c}0,69^{\star *} \\
0,39^{\star *} \\
-\end{array}$ & $\begin{array}{l}0,96^{\star \star} \\
0,95^{\star \star} \\
-\end{array}$ & $\begin{array}{c}0,95^{\star \star} \\
0,95^{\star \star} \\
-\end{array}$ & $\begin{array}{l}0,93^{\star \star} \\
0,95^{\star \star} \\
-\end{array}$ & \\
\hline $\mathrm{ESD} / \mathrm{TB}$ & $\begin{array}{l}0,61^{\text {**}} \\
0,44^{\star \star} \\
-\end{array}$ & $\begin{array}{l}0,44^{\star \star} \\
0,48^{\star \star} \\
-\end{array}$ & $\begin{array}{c}0,77^{\star *} \\
0,61^{* *} \\
-\end{array}$ & $\begin{array}{l}0,90^{\star *} \\
0,80^{* *} \\
-\end{array}$ & $\begin{array}{l}0,85^{\star \star} \\
0,72^{* \star} \\
-\end{array}$ & $\begin{array}{l}0,82^{\star \star} \\
0,74^{\star \star} \\
-\end{array}$ & $\begin{array}{l}0,87^{\star *} \\
0,68^{* *} \\
-\end{array}$ \\
\hline
\end{tabular}

* significatif (seuil $\mathbf{P}=0,05)$

* très significatif (seuil $\mathrm{P}=0,01$ ) 


\section{2) Etude statistique des relations entre le rendement en fromage et les principaux composants du lait}

Le tableau 2 représente la matrice des corrélations entre les différentes variables. Plusieurs remarques peuvent être faites sur les résultats rassemblés dans ce tableau.

- Les résultats obtenus diffèrent d'une laiterie à l'autre non seulement entre plusieurs types de fabrication mais également pour un même type de fromage.

- Il existe une corrélation très significative entre le taux de protéines, le taux de caséine isoélectrique, les matières azotées coagulables déterminées au laboratoire et les matières azotées coagulables déterminées d'après l'analyse de sérum recueilli en usine. Il existe également une corrélation significative entre ces mêmes valeurs et la teneur en substance sèche dégraissée du lait.

- Le rendement en fromage à $7 \mathrm{j}$ est fortement influencé par la richesse du lait en protéines, ou en caséine isoélectrique ou encore par les matières azotées coagulables, dans le cas de la laiterie C. Dans les deux laiteries A et B, aucun composant du lait ne semble influencer de façon très significative le rendement en fromage. En revanche, il existe une corrélation négative très significative entre le rendement et la teneur en substance sèche totale des fromages.

Dans le tableau 3, nous avons indiqué les corrélations obtenues entre les variables $Z_{1}-Z_{2}-Z_{2}^{\prime}$ d'une part et les variables TP/TB $\mathrm{TC} / \mathrm{TB}-\mathrm{MAC}_{\imath} / \mathrm{TB}-\mathrm{MAC}_{u} / \mathrm{TB}$ et ESD/TB d'autre part.

Pour la laiterie A, les corrélations obtenues entre le poids de fromage corrigé par $\mathrm{kg}$ de matière grasse (variable $\mathrm{Z}_{2}^{\prime}$ ) et les autres variables relatives aux constituants du lait sont nettement plus élevées que celles propres à la variable $\mathrm{Z}_{\text {. }}$. Pour la laiterie $\mathrm{B}$, les corrélations obtenues entre la variable $\mathrm{Z}_{2}{ }_{2}$ et les variables relatives aux constituants du lait sont au contraire inférieures à celles intéressant la variable $\mathrm{Z}_{2}$ sauf en ce qui concerne le rapport ESD/TB. Il en est de même pour la laiterie $C$ où les corrélations avec $Z_{2}^{\prime}$ sont inférieures à celles avec $Z_{2}$.

Pour déterminer l'influence respective des principaux éléments de la composition du lait sur le poids de fromage obtenu, nous avons effectué l'analyse de régression multiple (tab. 4 à 9) entre les variables exprimant le rendement d'une part (rendement à $\mathrm{J}+1$, à $\mathrm{J}+7$ et rendement corrigé à $\mathrm{J}+7$ pour les variations de teneur en eau des fromages) et 11 variables prises par 2, 3 ou 4 relatives au lait de fabrication et au fromage obtenu d'autre part.

Dans les tableaux 4, 5 et 6, nous avons calculé la signification des coefficients de régression pour tester l'importance des différentes variables dans les laiteries A - B - C. On peut remarquer :

- Laiteries A et B (tab. 4, 5 et 7). 
TABLEAU 4

Analyse de la régression multiple - Signification des coefficients (test $t^{2}$ ) - Laiterie A

\begin{tabular}{|c|c|c|c|c|c|c|c|c|c|c|c|c|c|c|c|c|c|c|c|c|}
\hline & $\mathrm{R}_{2}$ & $\mathrm{R}_{2}^{\prime}$ & $\mathrm{R}_{2}^{\prime}$ & $\mathrm{R}_{2}$ & $\mathrm{R}_{2}$ & $\mathrm{R}_{2}$ & $\mathrm{R}_{2}^{\prime}$ & $\mathrm{R}_{2}$ & $\mathrm{R}_{2}^{\prime}$ & $\mathrm{R}_{2}$ & $\mathrm{R}_{2}^{\prime}$ & $\mathrm{R}_{2}$ & $\mathrm{R}_{2}^{\prime}$ & $\mathrm{R}_{2}$ & $\mathrm{R}_{2}^{\prime}$ & $\mathrm{R}_{2}$ & $\mathrm{R}_{2}$ & $\mathrm{R}_{2}$ & $\mathrm{R}_{2}$ & $\mathrm{R}_{2}$ \\
\hline TP & 0,86 & $16,1^{* *}$ & & & & & & 1,6 & $16,2^{* *}$ & 0,8 & $20,2^{* *}$ & 2,0 & $15,6^{* *}$ & $17,7^{\star \star}$ & $18,4^{* *}$ & & & & $17,4^{* *}$ & \\
\hline $\begin{array}{l}\text { TB } \\
\text { TC }\end{array}$ & $7,65^{* *}$ & $84,6^{* *}$ & $68,4^{* *}$ & $6,5^{*}$ & & $8,8^{* *}$ & $76,4^{\star *}$ & $5,0^{*}$ & $117,6^{* \star}$ & $5,5^{*}$ & $111,4^{* *}$ & $7,1^{\star \star}$ & $106,0^{* *}$ & $101,6^{* *}$ & $102,4^{* *}$ & $80,9^{* *}$ & & $94,7^{* *}$ & $123,8^{* *}$ & $121,6^{* *}$ \\
\hline EST & & & & & $57,7^{* *}$ & & & & & & & & & & & & $65,9^{* *}$ & & & \\
\hline$\underset{f}{E S D}$ & & & & & 4 & $6,1^{*}$ & $4,7^{*}$ & & & & & & & 7601 1** & $193^{* *}$ & $5940^{* * *}$ & $4398^{\star *}$ & $\begin{array}{r}8,7^{* *} \\
6368^{* *}\end{array}$ & & $\begin{array}{r}11,3^{* *} \\
6708^{* *}\end{array}$ \\
\hline $\mathrm{TC} / \mathrm{TP}$ & & & & & 405,2 & & & $10,1^{\star \star}$ & $18,2^{\star \star}$ & & & & & 109,1 & 19,0 & $J 94,0$ & $5,4^{*}$ & & $10,0^{* *}$ & * \\
\hline & & & & & & & & & & & $15,3^{\star *}$ & $15,2^{* *}$ & $14,6^{* *}$ & & & & & & & \\
\hline test $F$ & $4^{*}$ & $51^{* \star}$ & $36^{* *}$ & $7^{* \star}$ & $282^{* *}$ & $7^{\star \star}$ & $40^{\star \star}$ & $7^{\star \star}$ & $48^{* *}$ & $6^{*}$ & $46^{\star *}$ & & & $288^{\star *}$ & $49^{\star *}$ & $240^{* *}$ & $201^{* *}$ & $258^{\star *}$ & $245^{\star *}$ & $227^{\star \star}$ \\
\hline $\begin{array}{l}\mathrm{R}^{2} \\
\mathrm{~s}^{\prime}{ }_{r}\end{array}$ & $\begin{array}{l}0,103 \\
0,742\end{array}$ & $\begin{array}{l}0,576 \\
0,235\end{array}$ & $\begin{array}{l}0,493 \\
0,257\end{array}$ & $\begin{array}{l}0,159 \\
0,719\end{array}$ & $\begin{array}{l}0,883 \\
0,268\end{array}$ & $\begin{array}{l}0,161 \\
0,717\end{array}$ & $\begin{array}{l}0,516 \\
0,251\end{array}$ & $\begin{array}{l}0,210 \\
0,701\end{array}$ & $\begin{array}{l}0,660 \\
0,212\end{array}$ & $\begin{array}{l}0,196 \\
0,708\end{array}$ & $\begin{array}{l}0,649 \\
0.215\end{array}$ & $\begin{array}{l}0,256 \\
0,680\end{array}$ & $\begin{array}{l}0,646 \\
0,216\end{array}$ & $\begin{array}{l}0,921 \\
0,221\end{array}$ & $\begin{array}{l}0,664 \\
0,210\end{array}$ & $\begin{array}{l}0,907 \\
0,241\end{array}$ & $\begin{array}{l}0,891 \\
0,261\end{array}$ & $\begin{array}{l}0,913 \\
0,233\end{array}$ & $\begin{array}{l}0,931 \\
0209\end{array}$ & $\begin{array}{l}0,926 \\
0216\end{array}$ \\
\hline$V_{2}$ & 75 & 75 & 75 & 75 & 75 & 75 & 75 & 74 & 74 & 74 & 74 & 74 & 74 & 74 & 74 & 74 & 74 & 74 & 73 & 73 \\
\hline
\end{tabular}

* significatif (seuil $\mathrm{P}=0,05)$

** très significatif (seuil $\mathrm{P}=0,01$ ) 
TABLEAU 5

Analyse de la régression multiple - Signification des coefficients (test $\left.\mathrm{t}^{2}\right)$ - Laiterie B

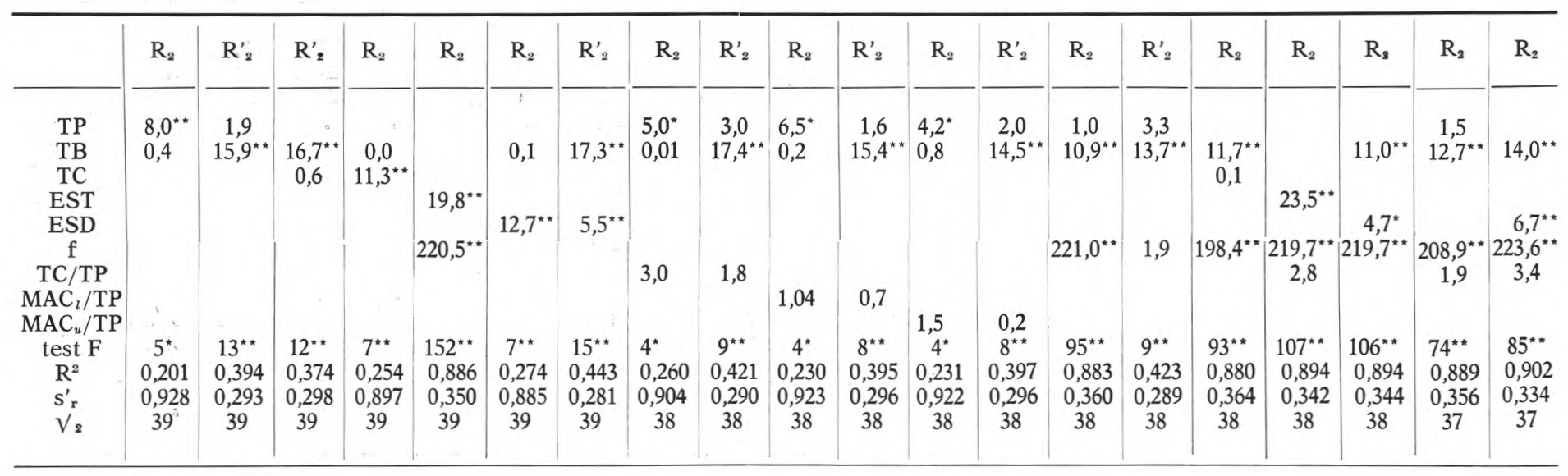

* significatif (seuil $\mathrm{P}=0,05)$

** très significatif (seuil $\mathrm{P}=0,01$ ) 
TABLEAU 6

Analyse de la régression multiple - Signification des coefticients (test $\mathrm{t}^{2}$ ) - Laiterie C

\begin{tabular}{|c|c|c|c|c|c|c|c|}
\hline & $\mathrm{R}_{2}$ & $\mathrm{R}_{2}^{\prime}$ & $\mathrm{R}_{2}$ & $\mathrm{R}_{2}^{\prime}$ & $\mathrm{R}_{2}$ & $\mathrm{R}_{2}$ & $\mathrm{R}_{2}^{\prime}$ \\
\hline $\mathrm{TP}$ & $122,0^{\star \star}$ & $20,0^{* *}$ & & & $155,4^{\star \star}$ & & \\
\hline TB & $166,7^{\star \star}$ & $50,7^{* *}$ & $53,9^{* *}$ & $44,8^{* *}$ & $70,3^{* *}$ & $75,4^{\star *}$ & $71,8^{\times *}$ \\
\hline $\mathrm{TC}$ & & & $129,9^{\star *}$ & $19,5^{\star \star}$ & & $83,6^{* \star}$ & $85,0^{\star \star *}$ \\
\hline $\mathrm{f}$ & & & & & $8,0^{* *}$ & $41,1^{\star *}$ & $86,4^{* *}$ \\
\hline test F & $122^{\star \star}$ & $45^{\star \star}$ & $128^{\star \star}$ & $45^{\star \star}$ & $89^{\star \star}$ & $128^{\star *}$ & $80^{* *}$ \\
\hline $\mathrm{R}^{2}$ & 0,672 & 0,432 & 0,682 & 0,429 & 0,692 & 0,764 & 0,671 \\
\hline$s_{r}^{\prime}$ & 0,324 & 0,360 & 0,319 & 0,361 & 0,315 & 0,276 & 0,276 \\
\hline$V_{2}$ & 119 & 119 & 119 & 119 & 118 & 118 & 118 \\
\hline
\end{tabular}

** très significatif (seuil $\mathrm{P}=0,01$ ) 
TABLEAU 7. - Equations de régression (laiterie A)

\begin{tabular}{|c|c|c|c|c|c|c|c|c|c|}
\hline $\mathrm{N}^{\circ}$ & & & & & & & $\mathrm{r}$ & $\begin{array}{c}\mathrm{R}^{2} \\
\text { en p. } 100\end{array}$ & $\begin{array}{l}\text { Ecart-type } \\
\text { résiduel } \\
\left(\mathrm{s}_{\mathrm{r}}^{\prime}\right)\end{array}$ \\
\hline 1 & $\mathrm{R}_{2}^{\prime}=0,1340$ & EST & $-2,111$ & & & & 0,60 & 36 & - \\
\hline 2 & $\mathrm{R}_{1}=0,2837$ & $\mathrm{TP}$ & $+0,2023$ & TB & & & 0,54 & 29 & - \\
\hline 3 & $\mathrm{R}_{1}=0,3877$ & TC & $\begin{array}{l} \\
+0,1822\end{array}$ & TB & & & 0,56 & 32 & - \\
\hline 5 & $\mathrm{R}_{1}=0,1349$ & ESD & $+0,1174$ & TB & & & 0,61 & 37 & - \\
\hline 6 & $\mathrm{R}_{2}=0,1835$ & $\mathrm{TP}$ & $\begin{array}{l}0,2702 \\
+\end{array}$ & TB & & & 0,37 & 14 & - \\
\hline 7 & $\mathrm{R}_{2}^{\prime}=0,1934$ & TP & $\begin{array}{r}0,2529 \\
+0\end{array}$ & $\mathrm{~TB}$ & & & 0,79 & 62 & - \\
\hline 8 & $\mathrm{R}_{2}=0,0895$ & TP & $+0,2095$ & $\mathrm{~TB}+$ & $\begin{array}{l}+5,058 \\
+\quad 5\end{array}$ & & 0,32 & 10 & 0,742 \\
\hline 9 & $\mathrm{R}_{2}^{\prime}=0,1223$ & TP & $+0,2203$ & $\mathrm{~TB}+$ & $\begin{array}{r}3,436 \\
+\quad 3\end{array}$ & & 0,76 & 58 & 0,235 \\
\hline 10 & $\mathrm{R}_{2}^{\prime}=0,2066$ & TC & $\begin{array}{r}0,2936 \\
+0\end{array}$ & $\mathrm{~TB}$ & & & 0,64 & 41 & - \\
\hline 11 & $\mathrm{R}_{2}^{\prime}=0,2275$ & $\mathrm{MAC}_{u}$ & $\begin{array}{r}0,2834 \\
+\end{array}$ & TB & & & 0,69 & 47 & - \\
\hline 12 & $\mathrm{R}_{2}^{\prime}=0,1974$ & $\mathrm{MAC}_{l}$ & $+0,3130$ & TB & & & 0,61 & 37 & - \\
\hline 13 & $\mathrm{R}_{2}^{\prime}=0,0799$ & ESD & $+0,2336$ & TB & & & 0,77 & 59 & - \\
\hline 16 & $\mathrm{R}_{2}=0,1470$ & EST & $-0,3141$ & $\mathrm{f}+$ & $+10,5072$ & & 0,94 & 88 & 0,268 \\
\hline 17 & $\mathrm{R}_{2}=0,1733$ & ESD & $+0,2171$ & TB - & $-\quad 7,9801$ & & 0,40 & 16 & 0,717 \\
\hline 18 & $\mathrm{R}_{2}^{\prime}=0,0528$ & ESD & $+0,2241$ & $\mathrm{~TB}+$ & $+\quad 1,7717$ & & 0,72 & 52 & 0,251 \\
\hline 19 & $\mathrm{R}_{2}=0,1164$ & $\mathrm{TP}$ & $+0,1631$ & $\mathrm{~TB}+$ & $+\quad 0,2100 \mathrm{TC} / \mathrm{TP}$ & $-10,8119$ & 0,46 & 21 & 0,701 \\
\hline 20 & $\mathrm{R}_{2}^{\prime}=0,1114$ & TP & $+0,2392$ & $\mathrm{~TB}$ & $-0,0854 \mathrm{TC} / \mathrm{TP}$ & $+\quad 9,8914$ & 0,81 & 66 & 0,212 \\
\hline 21 & $\mathrm{R}_{2}=0,0806$ & TP & $+0,1727$ & $\mathrm{~TB}+$ & $+0,1670 \mathrm{MAC}_{l} / \mathrm{TP}$ & $-5,7577$ & 0,44 & 20 & 0,708 \\
\hline 22 & $\mathrm{R}_{2}^{\prime}=0,1259$ & $\mathrm{TP}$ & $+0,2353$ & $\mathrm{~TB}$ & $-0,0680 \mathrm{MAC}_{l} / \mathrm{TP}$ & $+\quad 7,8432$ & 0,81 & 65 & 0,215 \\
\hline 23 & $\mathrm{R}_{2}=0,1241$ & $\mathrm{TP}$ & $+0,1852$ & $\mathrm{~TB}+$ & $+0,3286 \mathrm{MAC}_{u} / \mathrm{TP}$ & $-19,7796$ & 0,51 & 26 & 0,680 \\
\hline 24 & $\mathrm{R}_{2}^{\prime}=0,1116$ & TP & $+0,2279$ & $\mathrm{TP}$ & $-0,1023 \mathrm{MAC}_{u} / \mathrm{TP}$ & $+11,1729$ & 0,80 & 65 & 0,216 \\
\hline 25 & $\mathrm{R}_{2}=0,1211$ & TP & $+0,2278$ & $\mathrm{~TB}-$ & $-0,3322 \mathrm{f}$ & $+18,2654$ & 0,96 & 92 & 0,221 \\
\hline 26 & $\mathrm{R}_{2}^{\prime}=0,1176$ & TP & $+0,2176$ & $\mathrm{~TB}+$ & $+\quad 0,0500 \mathrm{f}$ & $+\quad 1,4469$ & 0,81 & 66 & 0,210 \\
\hline 27 & $\mathrm{R}_{2}=0,0682$ & $\mathrm{TC}$ & $+0,2231$ & $\mathrm{~TB}$ & $-0,3249 \mathrm{f}$ & $+20,4736$ & 0,95 & 91 & 0,241 \\
\hline 28 & $\mathrm{R}_{2}=0,1559$ & EST & $-0,3299$ & $\mathrm{f}-$ & $-0,0636 \mathrm{TC} / \mathrm{TP}$ & $+15,0620$ & 0,94 & 89 & 0,261 \\
\hline 29 & $\mathrm{R}_{2}=0,0681$ & ESD & $+0,2316$ & TB - & $-0,3242 \mathrm{f}$ & $+15,7506$ & 0,96 & 91 & 0,233 \\
\hline 30 & $\mathrm{R}_{2}=0,1138$ & TP & $+0,2443$ & TB - & $-\quad 0,3507 \mathrm{f}$ & $-0,0703 \mathrm{TC} / \mathrm{TP}+24,3093$ & 0,96 & 93 & 0,209 \\
\hline 31 & $\mathrm{R}_{2}=0,07205$ & ESD & $+0,25086$ & $\mathrm{~TB}$ & $-0,3447 \mathrm{f}$ & $-0,0820 \mathrm{TC} / \mathrm{TP}+22,1321$ & 0,96 & 93 & 0,216 \\
\hline
\end{tabular}


De toutes les variables examinées, celle qui apparaît jouer le plus grand rôle sur le rendement en fromage est la teneur en substance sèche du fromage. La prise en considération de cette variable dans une équation de régression multiple permet d'expliquer 88 p. 100 (équation 16 ) à 93 p. 100 (équations 30 et 31) des variations du rendement en fromage à $7 \mathrm{j}$, l'écart-type résiduel se réduisant à $0,268 \mathrm{~kg}$ (équation 16) voire même à $0,209 \mathrm{~kg}$ (équation 30 ) pour la laiterie $\mathrm{A}$, pour un rendement moyen $\mathrm{R}_{\mathrm{z}}$ égal à $14,3 \mathrm{~kg}$ de fromage aux $100 \mathrm{I}$ de lait; les écarts-type résiduels correspondants sont de $0,350 \mathrm{~kg}$ et $0,334 \mathrm{~kg}$ (équation 31) pour la laiterie $\mathrm{B}$, pour un rendement moyen $\mathrm{R}_{2}$ égal à $14,9 \mathrm{~kg} / 1001$.

De même, l'élimination des variations de teneur en eau des fromages par la formule de Maubois et Mocquot (1967) améliore très sensiblement l'estimation du rendement en fromage. Pour la laiterie $A$, en utilisant le couple des variables (TP et TB), le coefficient de corrélation multiple passe de 0,32 (équation 8 ) à 0,76 (équation 9), l'écart-type résiduel étant ramené de $0,742 \mathrm{~kg}$ à $0,235 \mathrm{~kg}$. Une amélioration de la précision est également obtenue avec les couples TC et TB (équations 14 et 15), ESD et TB (équations 17 et 18). Il en est de même pour la laiterie B.

La prise en considération du taux de caséine ou du taux de substance sèche dégraissée du lait au lieu du taux de protéines n'améliore pas considérablement l'estimation du rendement. Pour la laiterie A, la prise en considération du couple TP et TB explique 10 p. 100 des variations du rendement (équation 8) contre 16 p. 100 pour les couples TC/TB et ESD/TB ; l'écart-type résiduel passe respectivement de $0,742 \mathrm{~kg}$ à $0,719 \mathrm{~kg}$ et $0,717 \mathrm{~kg}$. Dans la laiterie $\mathrm{B}$, le couple TB/TP explique 20 p. 100 des variations du rendement contre 25 p. 100 pour le couple TC/TP et 27 p. 100 pour le couple ESD/TB, l'écart-type résiduel passant respectivement de $0,928 \mathrm{~kg}$ à $0,897 \mathrm{~kg}$ et $0,885 \mathrm{~kg}$.

La prise en considération dans une équation de régression multiple des variables exprimant soit la proportion de caséine précipitable à $\mathrm{pH} \mathrm{4,6}$ ( TC/TP), soit la proportion de matières azotées coagulées par la présure $\left(\mathrm{MAC}_{t} / \mathrm{TP} ; \mathrm{MAC}_{u} / \mathrm{TP}\right)$ dans les matières azotées totales, améliore l'estimation du rendement en fromage sensiblement de la même manière. Pour la laiterie $\mathrm{A}$, le coefficient de corrélation multiple passe de 0,32 (équation 8 ) à 0,46 avec TC/TP (équation 19), 0,44 avec $\mathrm{MAC}_{1} / \mathrm{TP}$ (équation 21) et 0,51 avec $\mathrm{MAC}_{u} / \mathrm{TP}$ (équation 23). Pour la laiterie B, ces corrélations sont respectivement de $0,45,0,51$, 0,48 et 0,48 , ce qui représente une très faible amélioration.

\section{- Laiterie C (tab. 6 et 9).}

Dans cette laiterie (qui fabrique un autre type de fromage, le Saint-Paulin) la prise en considération dans une équation de prédétermination du seul taux de protéines ou du taux de caséine permet d'expliquer plus de 50 p. 100 des variations du rendement en fromage (tab. 9, équations 1,2 et 3 ). La prise en considération du 
TABLEAU 8. - Equations de régression (laiterie B)

\begin{tabular}{|c|c|c|c|c|c|c|c|}
\hline $\mathrm{N}^{\circ}$ & & & & & $\mathrm{r}$ & $\begin{array}{c}\mathrm{R}^{2} \\
\text { en p. } 100\end{array}$ & $\begin{array}{c}\text { Ecart-type } \\
\text { résiduel } \\
\mathbf{s}^{\prime}{ }_{r}\end{array}$ \\
\hline 1 & $\mathrm{R}_{2}^{\prime}=0,1163 \mathrm{EST}$ & $-0,3313$ & & & 0,61 & 37 & - \\
\hline 2 & $\mathrm{R}_{1}=0,4841 \mathrm{TP}$ & $-0,0117$ ТВ & & & 0,49 & 24 & - \\
\hline 3 & $\mathrm{R}_{1}=0,6358 \mathrm{TC}$ & $-0,0244$ TB & & & 0,54 & 29 & - \\
\hline 4 & $\mathrm{R}_{1}=0,6252 \mathrm{MAC}_{u}$ & $+0,0120$ TB & & & 0,48 & 23 & - \\
\hline 5 & $\mathrm{R}_{1}=0,1779 \mathrm{ESD}$ & $+0,0049$ TB & & & 0,44 & 20 & - \\
\hline 6 & $\mathrm{R}_{2}=0,4575 \mathrm{TP}$ & $-0,0267$ TB & & & 0,46 & 21 & - \\
\hline 7 & $\mathrm{R}_{2}^{\prime}=0,1592 \mathrm{TP}$ & $+0,2867 \mathrm{~TB}$ & & & 0,49 & 24 & - \\
\hline 8 & $\mathrm{R}_{2}=0,5027 \mathrm{TP}$ & $+0,0372$ TB & $-3,3256$ & & 0,45 & 20 & 0,928 \\
\hline 9 & $\mathrm{R}_{2}^{\prime}=0,0770 \mathrm{TP}$ & $+0,2378 \mathrm{~TB}$ & $+\quad 4,1674$ & & 0,63 & 39 & 0,293 \\
\hline 10 & $\mathrm{R}_{2}^{\prime}=0,1365 \mathrm{TC}$ & $+0,3519$ ТВ & & & 0,36 & 13 & - \\
\hline 11 & $\mathrm{R}_{2}^{\prime}=0,1641 \mathrm{MAC}_{u}$ & $+0,3327$ TB & & & 0,39 & 15 & - \\
\hline 12 & $\mathrm{R}_{2}^{\prime}=0,1709 \mathrm{MAC}_{l}$ & $+0,3274$ TB & & & 0,43 & 18 & - \\
\hline 13 & $\mathrm{R}_{2}^{\prime}=0,0792 \mathrm{ESD}$ & $+0,2249$ TB & & & 0,61 & 37 & - \\
\hline 14 & $\mathrm{R}_{2}^{\prime}=0,0443 \mathrm{TC}$ & $+0,2500 \mathrm{~TB}$ & $+\quad 5,2781$ & & 0,61 & 37 & 0,298 \\
\hline 15 & $\mathrm{R}_{2}=0,5959 \mathrm{TC}$ & $-0,0075$ TB & $-\quad 0,7454$ & & 0,50 & 25 & 0,897 \\
\hline 16 & $\mathrm{R}_{2}=0,1327 \mathrm{EST}$ & $-0,3600 \mathrm{f}$ & $+14,0871$ & & 0,94 & 89 & 0,350 \\
\hline 17 & $\mathrm{R}_{2}=0,3414 \mathrm{ESD}$ & $+0,0561 \mathrm{~TB}$ & $-17,5179$ & & 0,52 & 27 & 0,885 \\
\hline 18 & $\mathrm{R}_{2}^{\prime}=0,0711 \mathrm{ESD}$ & $+0,2308 \mathrm{~TB}$ & $+\quad 0,5698$ & & 0,66 & 44 & 0,281 \\
\hline 19 & $\mathrm{R}_{2}=0,4073 \mathrm{TP}$ & $-0,0077 \mathrm{~TB}$ & $+\quad 0,2757 \mathrm{TC} / \mathrm{TP}$ & $-20,2608$ & 0,51 & 26 & 0,904 \\
\hline 20 & $\mathrm{R}_{2}^{\prime}=0,1004 \mathrm{TP}$ & $+0,2488 \mathrm{~TB}$ & $-0,0675 \mathrm{TC} / \mathrm{TP}$ & $\begin{array}{r}8,3127 \\
+\quad 8,2\end{array}$ & 0,65 & 42 & 0,290 \\
\hline 21 & $\mathrm{R}_{2}=0,4602 \mathrm{TP}$ & $+0,0243 \mathrm{~TB}$ & $+0,1758 \mathrm{MAC}_{l} / \mathrm{TP}$ & $-14,4924$ & 0,48 & 23 & 0,923 \\
\hline 2.2 & $\mathrm{R}_{2}^{\prime}=0,0739 \mathrm{TP}$ & $+0,2368 \mathrm{~TB}$ & $+0,0128 \mathrm{MAC}_{l} / \mathrm{TP}$ & $+\quad 3,3540$ & 0,63 & 40 & 0,296 \\
\hline 23 & $\mathrm{R}_{2}=0,4017 \mathrm{TP}$ & $-0,0564 \mathrm{~TB}$ & $+\quad 0,2853 \mathrm{MAC}_{u} / \mathrm{TP}$ & $-18,4519$ & 0,48 & 23 & 0,922 \\
\hline 24 & $\mathrm{R}_{2}^{\prime}=0,0885 \mathrm{TP}$ & $+0,2484$ TB & - $0,0325 \mathrm{MAC}_{u} / \mathrm{TP}$ & $\begin{array}{r}0,8926 \\
+\quad 5\end{array}$ & 0,63 & 40 & 0,296 \\
\hline 25 & $\mathrm{R}_{2}=0,0764 \mathrm{TP}$ & $+0,2465$ ТВ & $-0,3846 \mathrm{f}$ & $+21,3197$ & 0,94 & 88 & 0,360 \\
\hline 26 & $\mathrm{R}_{2}^{\prime}=0,1092 \mathrm{TP}$ & $+0,2219$ ТВ & $+\quad 0,0290 \mathrm{f}$ & $+\quad 2,3053$ & 0,65 & 42 & 0,289 \\
\hline 27 & $\mathrm{R}_{2}=0,0297 \mathrm{TC}$ & $+0,2651 \mathrm{~TB}$ & $-0,3899 \mathrm{f}$ & $+22,8441$ & 0,94 & 88 & 0,364 \\
\hline 28 & $\mathrm{R}_{2}=0,1516 \mathrm{EST}$ & $-0,3747 \mathrm{f}$ & $-0,1096 \mathrm{TC} / \mathrm{TP}$ & $+20,9932$ & 0,95 & 89 & 0,342 \\
\hline 29 & $\mathrm{R}_{2}=0,0891 \mathrm{ESD}$ & $+0,2285 \mathrm{~TB}$ & $-0,3720 \mathrm{f}$ & $+15,8654$ & 0,94 & 89 & 0,344 \\
\hline 30 & $\mathrm{R}_{2}=0,0927 \mathrm{TP}$ & $+0,2697 \mathrm{~TB}$ & $-0,3992 \mathrm{f}$ & $-0,0940 \mathrm{TC} / \mathrm{TP}+28,0303$ & 0,94 & 89 & 0,356 \\
\hline 31 & $\mathrm{R}_{2}=0,1057 \mathrm{ESD}$ & $+0,2568 \mathrm{~TB}$ & $-0,3889 \mathrm{f}$ & $-0,1178 \mathrm{TC} / \mathrm{TP}+23,4343$ & 0,95 & 90 & 0,334 \\
\hline
\end{tabular}


TABLEAU 9. - Equations de régression (laiterie $\mathrm{C}$ )

\begin{tabular}{|c|c|c|c|c|c|}
\hline $\mathrm{N}^{\circ}$ & & & $\mathrm{r}$ & $\begin{array}{c}R^{2} \\
\text { en p. } 100\end{array}$ & $\mathrm{~S}_{\mathrm{r}}^{\prime}$ \\
\hline 1 & $\mathrm{R}_{2}=0,3916 \mathrm{TP}$ & $-1,6863$ & 0,70 & 49 & - \\
\hline 2 & $\mathrm{R}_{2}=0,4112 \mathrm{TC}$ & $+0,5684$ & 0,73 & 54 & - \\
\hline 3 & $\mathrm{R}_{2}=0,4283 \mathrm{MAC}_{l}$ & $+0,6966$ & 0,71 & 50 & - \\
\hline 4 & $\mathrm{R}_{2}=0,2214 \mathrm{TP}$ & $+0,1636$ ТВ & 0,73 & 54 & 一 \\
\hline 5 & $\mathrm{R}_{2}^{\prime}=0,1764 \mathrm{TP}$ & $+0,2240$ ТВ & 0,64 & 41 & - \\
\hline 6 & $\mathrm{R}_{2}=0,2857 \mathrm{TC}$ & $+0,1557 \mathrm{~TB}$ & 0,78 & 60 & 一 \\
\hline 7 & $\mathrm{R}_{2}^{\prime}=0,2088 \mathrm{TC}$ & $+0,2379$ TB & 0,62 & 39 & - \\
\hline 8 & $\mathrm{R}_{2}=0,3342 \mathrm{TP}$ & $+0,2011$ TB $-4,9216$ & 0,82 & 67 & 0,324 \\
\hline 9 & $\mathrm{R}_{2}^{\prime}=0,1508 \mathrm{TP}$ & $+0,1952$ TB $+1,6462$ & 0,66 & 43 & 0,360 \\
\hline 10 & $\mathrm{R}_{2}=0,3455 \mathrm{TC}$ & $+0,1811$ TВ $-2,3261$ & 0,82 & 68 & 0,319 \\
\hline 11 & $\mathrm{R}_{2}^{\prime}=0,1516 \mathrm{TC}$ & $+0,1872$ TB $+2,9087$ & 0,66 & 43 & 0,361 \\
\hline 12 & $\mathrm{R}_{2}=0,3299 \mathrm{TP}$ & $+0,2007$ ТВ $-0,0189 \mathrm{f}-3,8550$ & 0,83 & 69 & 0,315 \\
\hline 13 & $\mathrm{R}_{2}^{\prime}=0,1564 \mathrm{TP}$ & $+0,1957$ TB $+0,0246 \mathrm{f}+0,2586$ & 0,69 & 48 & 0,346 \\
\hline 14 & $\mathrm{R}_{2}=0,2655 \mathrm{TC}$ & $+0,1855$ TВ $-0,1114 \mathrm{f}+5,1603$ & 0,87 & 76 & 0,276 \\
\hline 15 & $\mathrm{R}_{2}^{\prime}=0,2677 \mathrm{TC}$ & $+0,1809$ TВ $+0,1616 \mathrm{f}-7,9503$ & 0,82 & 67 & 0,276 \\
\hline
\end{tabular}


taux butyreux et du taux de protéines dans une équation de régression multiple explique 67 p. 100 de ces variations (équation 8) (tab. 9). L'introduction du taux de substance sèche du fromage en plus de ces deux variables explique seulement 69 p. 100 des variations du rendement, résultat inférieur à celui obtenu dans les laiteries A et B avec le même type d'équation. Le remplacement dans ce dernier type d'équation du taux de protéines par le taux de caséine permet d'expliquer 76 p. 100 des variations du rendement. L'élimination des variations de teneur en eau des fromages n'améliore pas l'estimation du rendement en fromage, contrairement à ce qui se passe pour les deux autres laiteries.

\section{DISCUSSION}

L'incidence des variations de teneur en eau des fromages sur le rendement (de la transformation) du lait en fromage est si importante, dans le cas des fromages du type Camembert, qu'elle " masque » le rôle joué par les deux constituants principaux du lait : protéines ou graisse. L'industriel fabriquant des fromages de type pâte molle doit donc uniformiser au mieux ses fabrications (en ce qui concerne le poids moyen et la teneur en substance sèche) non seulement pour diminuer les marges de sécurité (qui autrement seraient indispensables) mais également pour mieux estimer la quantité d'un fromage de composition définie qui peut être obtenue avec le lait qu'il reçoit.

Le rôle joué par le taux de protéines et le taux de graisse dans l'estimation du rendement en fromage est mis en évidence lorsqu'on élimine ces variations de teneur en eau des fromages, soit en introduisant cette dernière variable dans une équation de régression multiple, soit en ramenant par le calcul toutes les fabrications à la même teneur en substance sèche selon Maubois et Mocquot (1967). On doit remarquer que la première solution donne une meilleure estimation du rendement que la seconde. Cela est probablement dû au fait que, dans l'établissement de notre formule, nous avons supposé que :

1) Les fromages analysés ne subissaient aucune perte de poids par évaporation. Or tel n'est pas le cas dans la pratique fromagère où les fromages sont souvent placés dans une atmosphère qui permet une certaine dessiccation.

2) Le lactosérum inclus dans les fromages avait la même composition que la totalité du lactosérum recueilli au cours de l'égouttage. Or nous avons montré d'autre part (Maubois et Mocquot, 1969) que la teneur en substance sèche du lactosérum s'égouttant d'un caillé destiné à la fabrication d'un fromage du type pâte molle variait dans des proportions non négligeables.

Toutefois, on peut aussi remarquer qu'après correction des variations de la teneur en eau des fabrications, les coefficients de régres- 
sion des équations de prédétermination établis dans les laiteries A et B sont assez peu différents (équations $7,9,10,11,12,13$ des tab. 7 et 8 ). Les écarts qui subsistent sont probablement à imputer aux différences de techniques de fabrication existant entre les deux laiteries (moulage à la louche dans un cas, au multimoule dans l'autre). L'examen des coefficients de régression montre qu'à extrait sec égal le poids de fromage obtenu dans la laiterie $\mathrm{B}$ à partir d'un lait ayant une composition définie est plus faible que celui obtenu dans la laiterie A. Le moulage au multimoule provoque donc une perte de rendement.

Nos résultats montrent que l'estimation du rendement en fromage à partir du taux de caséine, du taux de matières azotées coagulables ou du taux de substance sèche dégraissée du lait n'est pas sensiblement meilleure que celle obtenue à partir du taux de matières azotées totales. Cela signifie que la "valeur fromagère " d'un lait s'apprécie avec une précision suffisante dans une région déterminée et avec une technique de fabrication déterminée, par la seule détermination du taux de protéines du lait. Ceci confirme les observations de Mocquot et al. (1963) sur la faible variation du rapport Matières Azotées Coagulables / Matières Azotées Totales.

Dans une fabrication à technologie bien établie et maîtrisée, comme celle du Saint-Paulin (cas de la laiterie C), près de 50 p. 100 des variations du rendement en fromage sont explicables par la variation du seul taux de protéines. La maîtrise totale des variations de teneur en eau permettrait à l'industriel fabriquant du Saint-Paulin de prévoir jusqu'à 75 p. 100 des variations du rendement en fromage en tenant compte du taux de caséine et du taux de graisse du lait de fabrication.

Nos résultats ont été obtenus sur du lait traité dans les conditions de fabrication en usine. Ils sont directement applicables à une prédétermination quotidienne des rendements dans les usines travaillant selon les techniques de fabrication que nous avons décrites.

Cette prédétermination, peut se faire en utilisant les résultats statistiques de notre étude, de la manière suivante :

1) L'industriel fromager détermine la teneur en protéines du lait de fabrication (TP).

2) Il ajuste la teneur en graisse (TB) de ce lait en utilisant la relation moyenne existant entre ces deux variables pour la fabrication concernée. Par exemple, dans le cas de la laiterie A, TB $=0,8206$ TP pour obtenir un fromage de type Camembert ayant un rapport matière grasse dans la matière sèche totale de 46,8 à $\mathrm{J}+7$ (tab, 1).

3) Une bonne prédétermination du poids de fromage qui peut être obtenue avec ce lait de fabrication ne peut être calculée que si l'industriel fromager contrôle parfaitement la teneur en substance sèche de ses fabrications. Comme avec la technologie actuelle de fabrication des fromages du type Camembert, un tel contrôle est impossible, une estimation du rendement en fromage sera obtenue avec une bonne précision $(\mathrm{r}=0,96)$, en introduisant dans l'équation 
25 (tab. 7) outre le TP et le TB précédemment déterminés, un terme "f " (teneur en substance sèche du fromage) qui sera, soit la teneur en substance sèche de la fabrication réalisée au jour J-1, soit la teneur en substance sèche moyenne des fabrications réalisées aux jours $\mathrm{J}-1$; J-2 ; J-3.

Ces résultats illustrent les possibilités réelles offertes à l'industriel fromager pour améliorer la qualité de son travail. Nous examinerons, dans une prochaine étude, les modifications à apporter aux équations de régression, dans le cas d'usines utilisant d'autres techniques de fabrication.

\section{Remerciements}

Nous tenons à remercier Mme Triballat, MM. Nazart et Roché pour l'aide qu'ils nous ont constamment apportée lors de nos expérimentations dans leurs usines respectives, Nous tenons également à remercier $M$. Sainclivier pour ses suggestions dans l'élaboration et la réalisation de ce travail qui est la continuation de celui qu'il avait entrepris (Mocquot et al., 1963).

\section{$R$ és u m é}

Les paramètres caractéristiques de 120 fabrications de Camembert, en vraie grandeur (10000 1 chacune) et de 122 fabrications de Saint-Paulin (4800 I chacune) ont été déterminés et analysés statistiquement. Les résultats obtenus montrent que l'estimation du " rendement en fromage " d'un lait de fabrication à partir de l'analyse des constituants de ce lait ne peut se faire avec une bonne précision, qu'en éliminant les variations de teneur en eau des fromages. Cette élimination peut se faire soit en introduisant ce dernier paramètre dans une équation de régression multiple, soit en ramenant toutes les fabrications à la même teneur en substance sèche par le calcul. Une méthode de prédétermination du rendement en fromage est proposée à partir des résultats statistiques de notre étude.

\section{S u m m a ry}

The cheese yielding capacity of milk in Camembert and Saint-Paulin Cheesemaking

A study was undertaken on a factory scale in three dairies situated in Brittany; two making Camembert cheese ; one making SaintPaulin Cheese.

$1^{\circ}$ To determine the milk, whey and cheese composition concerning total solids, fat, total proteins, casein (isoelectric and rennet) over two and a half year.

$2^{\circ}$ To determine by the method of multiple regression, the respective influence of these constituents on the cheese yield (weight of cheese obtained from $100 \mathrm{l}$ of milk). 
The results show that the cheese yielding capacity of milk in soft type cheese can be estimated accurately only when the variations in moisture content of the cheese are eliminated. This can be donc :

a) by introducing the cheese moisture content in a multiple regression equation (in this case, about 92 per cent of the variations in cheese yield can be explained from the fat and protein content of the milk) or,

b) by assuming that in all the experiments the cheese should have had the same moisture content according to the calculation of Maubois and Mocquot (1967).

A method for the estimation of the cheese yield from the actual milk composition based on the statistical results of this study is proposed.

Reçu pour publication en avril 1970.

\section{Références bibliographiques}

[1] Aschaffenburg (R.), Drewry (J.) (1959). - New procedure for the routine determination of the various non-casein proteins of milk. $X V^{m e}$ Congr. Intern. Laiterie, Londres, 3, 1631-1637.

[2] Mc Dowali. (F. M.) (1936). - The cheese yielding capacity of milk and its relation to the method of payment for milk for cheesemaking. Dairy Res. Inst., N.Z., 72, 137-364

[3] Maubois (J. L.), Mocouot (G.) (1969). - Résultats non publiés.

[4] Maubois (J. L.), Mocouot (G.) (1967). - Comment ramener à la même teneur en substance sèche des fabrications de fromage en vue de comparer les "rendements " respectifs du lait en fromage. Rev. Lait Franç, 239, 15-18.

[5] Mocouot (G.), Ricordeau (G.), Auriol (P.), (1963). - Estimation du rendement en fromage de gruyère de Comté en fonction de la richesse du lait de chaudière. Ann. Zootech., 12 (1), 53-66.

[6] Mocouot (G.), Blanc-Patin (E.), Sainclivier (M.), Rousseaux (P.), Jeunet (R.) (1963), - Relation entre la teneur du lait en matière azotée et le poids de fromage obtenu. Ind. Lait., 203, 269-276.

[7] Portmann A., Pierre Alice, Vedrenne (P.) (1968). - Relation entre teneur en matière grasse et azotée du lait de chèvre et rendement en fromage. Rev. Lait. Franç., 251, 97-101.

[8] Ricordeau (G.), Mocouot (G.) (1967). - Influence des variations saisonnières de la composition du lait de chèvre sur le rendement en fromage. Conséquences pratiques pour la sélection. Ann. Zootech., 16 (2), 165-181.

[9] Schulz (M. E.), KaY (H.) (1957). - Käse-Tabellen : Eiweisstiter, Feit. teinstellung, Käsesollaus bente. Milchw. Verlag Th. Mann. K. G. Hildesheim.

[10] SHELtoN A. B. (1945). - Cheddar cheese composition and yield in relation to fat and casein content of milk. Publication of N.S.W. Dept. of Agriculture. Division of Dairying issue.

[11] Van Slyke (L. L.), Price (W. V.) (1952). - Cheese, Orange Iudd. Pub. Co., New-York. 